\title{
Erratum: Factors that affect the onset of action of non-depolarizing neuromuscular blocking agents
}

\author{
Yong Beom Kim ${ }^{1}$, Tae-Yun Sung ${ }^{2}$, and Hong Seuk Yang ${ }^{3}$ \\ Department of Anesthesiology and Pain Medicine, ${ }^{1}$ Gil Medical Center, Gachon University College of Medicine, \\ Incheon, ${ }^{2}$ Konyang University Hospital, Konyang University College of Medicine, Daejeon, ${ }^{3}$ Asan Medical Center, \\ University of Ulsan College of Medicine, Seoul, Korea
}

Korean J Anesthesiol 2017 October 70(5): 500-510

The article by Kim YB, et al. entitled, "Factors that affect the onset of action of non-depolarizing neuromuscular blocking agents" (Korean J Anesthesiol 2015 Oct 70(5): 500-510) contained an error in the text and author's name.

Before correction:

Page 504, Statins (3-hydroxy-3-methylglutaryl coenzyme A reductase inhibitors)

Statins decrease the risk of cardiovascular disease by decreasing low-density lipoprotein cholesterol (LDL-C) levels. However, statins increase muscle creatine kinase (CK) with muscle toxicity.

With long-term administration, the onset time of NMBDs is delayed and the duration of action is reduced [78,79].

The correct information is found below:

Page 504, Statins (3-hydroxy-3-methylglutaryl coenzyme A reductase inhibitors)

Statins decrease the risk of cardiovascular disease by decreasing low-density lipoprotein cholesterol (LDL-C) levels. However, statins increase muscle creatine kinase (CK) with muscle toxicity.

With long-term administration, the onset time of NMBDs is shortened and the duration of action is prolonged [78,79]

And the first author's name was misspelled as Yong Byum Kim. The correct spelling is Yong Beom Kim.

The authors apologize for any inconvenience this mistake may have caused.

Korean J Anesthesiol 2017 December 70(6): 656

https://doi.org/10.4097/kjae.2017.70.6.656

(c) This is an open-access article distributed under the terms of the Creative Commons Attribution Non-Commercial License (http://creativecommons.org/ licenses/by-nc/4.0/), which permits unrestricted non-commercial use, distribution, and reproduction in any medium, provided the original work is properly cited. 